\title{
Gouy phase shift of single-cycle picosecond acoustic pulses
}

\author{
N. C. R. Holme, B. C. Daly, M. T. Myaing, and T. B. Norris ${ }^{a)}$ \\ Center for Ultrafast Optical Science and EECS Department, University of Michigan, 2200 Bonisteel Blvd., \\ Ann Arbor, Michigan 48109-2099
}

(Received 20 June 2002; accepted 15 May 2003)

\begin{abstract}
Ultrafast laser pulses are used to generate single-cycle picosecond acoustic pulses in thin metal films on silicon. For small initial excitation spot sizes, propagation of the acoustic pulses across a $485-\mu \mathrm{m}$ $\mathrm{Si}$ crystal leads to significant diffraction effects. The temporal reshaping of the acoustic wave form due to diffraction is investigated, and we demonstrate that the acoustic far field can be reached.
\end{abstract}

(C) 2003 American Institute of Physics. [DOI: 10.1063/1.1590405]

The generation of picosecond-duration acoustic pulses through the use of pico- or femtosecond lasers, and the application of these acoustic pulses to the study of materials and thin-film structures, has become a powerful and widely used technique. ${ }^{1-3}$ When an ultrashort optical pulse is used to heat a thin transducer layer (such as aluminum) on a crystal substrate, an acoustic pulse shorter than 10 ps can be launched into the substrate. One of the principal applications of picosecond ultrasonics has been to characterize materials and interfaces in multilayer systems. ${ }^{1-3}$ The basic concept is time-domain reflectometry or one-dimensional imaging, where the reflection of the acoustic pulse from each interface is measured; this yields information such as the film thickness, frequency-dependent acoustic attenuation, etc. In the work reported so far, the transverse width of the acoustic source (determined by the laser spot size) has been large, or the propagation length short, such that the acoustic propagation may be considered to be essentially that of a plane wave.

In this letter, we consider the case in which the excitation laser beam is tightly focused, and the acoustic pulse is allowed to propagate a sufficiently long distance that the acoustic pulse diffracts; when the pulse has propagated to the far field, the pulse temporal profile becomes that of the temporal derivative of the input pulse wave form as a result of the Gouy phase shift. The observation of propagationinduced pulse shape changes is important for understanding both linear and nonlinear picosecond ultrasonics applications, in which diffraction cannot be neglected. More importantly, it opens up the possibility of performing highresolution two-dimensional acoustic imaging with singlecycle pulses using reconstruction algorithms demonstrated elsewhere. ${ }^{4}$

In our experiments, a 15-nm layer of aluminum is deposited on a silicon substrate. When a short (100-fs) laser pulse is incident on this layer, transient heating of the layer results in the generation of a few-picosecond-duration acoustic pulse, which propagates into the substrate. The initial shape of the pulse is determined by the optical absorption length and speed of sound $v$ in the $\mathrm{Al} \mathrm{film.}{ }^{3}$ Due to the absorption of very high frequency phonons, the acoustic pulse shape can be described to a good approximation by a function of the form $u_{o}(\tau) \propto \tau e^{-\tau^{2} / 2}$, where $\tau=(t-z / v) / t_{o}$ is the local time

${ }^{a)}$ Electronic mail: tnorris@eecs.umich.edu normalized to the pulse width $2 t_{o}, z$ is the longitudinal position, and $v$ the velocity.

As this single-cycle acoustic pulse propagates into the substrate, diffraction causes the pulse shape to change. Diffraction-induced pulse shape changes have been studied in some detail for picosecond-duration electromagnetic pulses: Ruffin et al. ${ }^{5}$ have observed that as a single-cycle pulse propagates through a focus, the polarity of the pulse flips sign, and have shown that this is a manifestation of the Gouy phase shift. ${ }^{6}$ In general, the diffraction of a monochromatic wave is accompanied by a $\pi / 2$ phase shift between the near-field and far-field wave forms; this is described mathematically by the $-i \omega / c$ factor that appears in front of the Huygens-Fresnel diffraction formula. ${ }^{7}$ For broadband diffracting fields, the Huygens-Fresnel diffraction formula may be Fourier transformed to the time domain, with the result that the time-domain far-field wave form is given by the temporal derivative of the input wave form: ${ }^{8}$

$$
u\left(P_{0}, t\right)=\iint_{\Sigma} \frac{\cos \theta}{2 \pi c r_{01}} \frac{\partial}{\partial t} u\left(P_{1}, t-\frac{r_{01}}{c}\right) d \sigma .
$$

To describe quantitatively the pulse shape changes which occur on diffraction, we follow the treatment developed by Kaplan, describing the evolution of an initial pulse given by $E_{z=0}(r, \tau)=E_{o} \tau e^{-\tau^{2} / 2} e^{-r^{2} / 2 r_{o}^{2}{ }^{9}}$ For a single-cycle pulse of duration $2 t_{o}$ and transverse beam size $2 r_{o}$, the characteristic diffraction length is $z_{d}=r_{o}^{2} / v t_{o}$. We define a normalized propagation length $\zeta=z / z_{d}$. For this initial pulse shape, the general form of the field on axis is

$$
\begin{aligned}
u_{\text {on }} \propto & e^{-\tau^{2} / 2}\left(\zeta+\tau-\zeta^{2} \sqrt{\pi / 2} e^{(\tau-\zeta)^{2} / 2}\{1+\operatorname{erf}[(\tau\right. \\
& -\zeta) / \sqrt{2}]\}) .
\end{aligned}
$$

When the propagation distance is much larger than $z_{d}$, that is, $\zeta \rightarrow \infty$, so that the pulse is in the far-field limit, then the pulse shape becomes essentially the temporal derivative of the input: $u_{\text {on }}(\tau, \zeta) \rightarrow \zeta^{-1} \partial u_{o}(\tau) / \partial \tau$. As discussed earlier, this change in shape is the result of the $\pi / 2$ Gouy phase shift that occurs in propagation from the near to the far field.

For $\langle 111\rangle$ silicon, with a longitudinal acoustic velocity $v=9.4 \times 10^{3} \mathrm{~m} / \mathrm{s}$, a single-cycle pulse of duration $t_{o}=2.5 \mathrm{ps}$ will have a spectrum with a peak wavelength $\lambda_{\text {peak }} \sim 120 \mathrm{~nm}$ and a peak frequency of $\sim 80 \mathrm{GHz}$. For a spatial waist $r_{o}=3.25 \mu \mathrm{m}$ the characteristic diffraction length is $z_{d}=450$ 

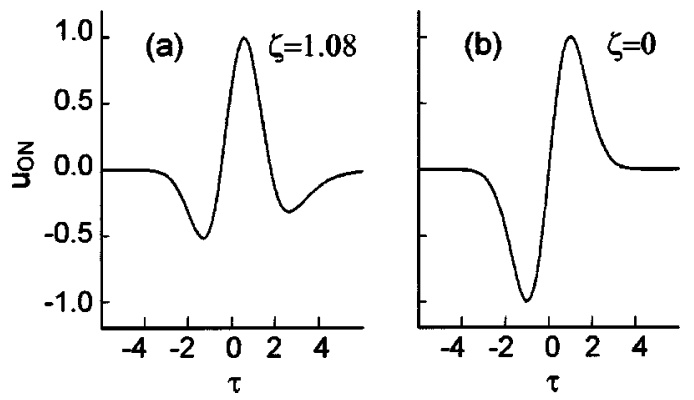

FIG. 1. Acoustic wave forms as calculated from Eq. (2). Results are shown for (a) the far field $(\zeta=1.08)$ and (b) the near field $(\zeta=0)$ of the source. The calculation is plotted versus normalized time $\tau=(t-z / v) / t_{o}$ as described in the text.

$\mu \mathrm{m}$. Thus, if the pulse propagates across a $485-\mu \mathrm{m} \mathrm{Si}$ wafer, $\zeta=1.08$, and thus the pulse shape should evolve from a single-cycle to nearly a derivative shape. The calculated pulse shapes for this case and the case of $\zeta=0$ (near-field limit) are shown in Fig. 1. In order to observe the effects of diffraction on picosecond ultrasonic pulses, we measured the pulse shape after propagation across a fixed length of 485 $\mu \mathrm{m}$. We varied the parameter $\zeta$ by adjusting the focal spot size of the excitation laser beam, thereby changing the initial waist size $r_{o}$ of the acoustic source.

The picosecond ultrasonic pulses were generated and measured using the femtosecond optical pump-probe setup shown in Fig. 2. The laser was a home-built mode-locked Ti:sapphire oscillator operating at $830 \mathrm{~nm}$, with a pulse duration of $100 \mathrm{fs}$ and repetition rate of $86 \mathrm{MHz}$. The sample was a double-side polished wafer of high-purity (nominally undoped) $\langle 111\rangle \mathrm{Si}$, with 15-nm Al transducer layers deposited on front and back surfaces. The laser was split into pump and probe beams, and the pump was acousto-optically modulated at $\sim 1 \mathrm{MHz}$ and focused onto the front surface of the sample. The probe was sent through an optical delay line and focused onto the back surface, and the modulated reflectivity monitored with a photodetector and lock-in amplifier; the noise limit of the differential reflectivity measurement was $<10^{-6}$. The sample was held at a temperature of $10 \mathrm{~K}$ in a cryostat to reduce the acoustic phonon losses.

Due to the large difference between the speed of sound and the speed of light, the acoustic propagation through 485 $\mu \mathrm{m}$ of $\mathrm{Si}$ requires an optical delay of $15 \mathrm{~m}$; thus, in order to obtain arbitrary optical delays, a delay line as long as the laser cavity was built. Since the optical probe beam must be aligned to be coaxial with the acoustic pulse with submicron

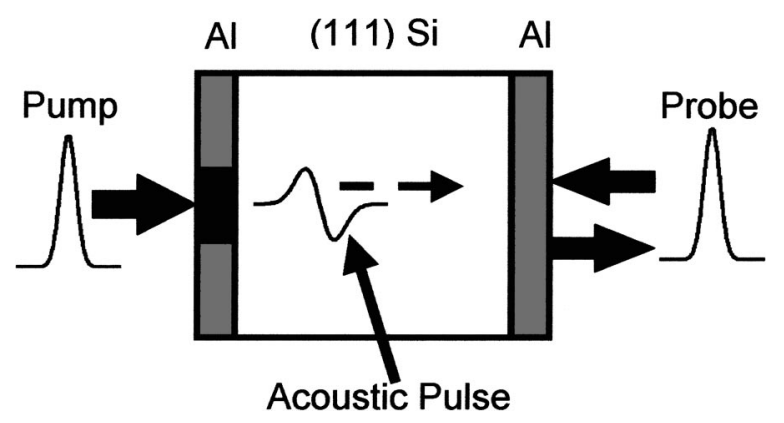

FIG. 2. Experimental configuration. 100-fs optical pump and probe pulses were focused onto the front and back surfaces, respectively, allowing the propagation of the acoustic wave across the wafer to be measured.

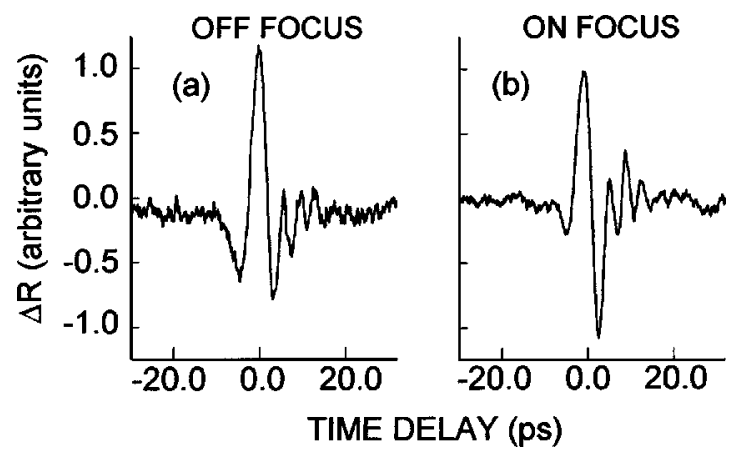

FIG. 3. Acoustic wave forms measured for weak (left) and tight (right) focusing of the excitation beam: (a) the signal on the left corresponds to the near-field acoustic pulse shape $(\zeta=0.04)$ and (b) that on the right corresponds to the far field $(\zeta=1.08)$.

accuracy, a beam pointing stabilizer was built to maintain high alignment accuracy over the entire optical delay. The sample and probe focusing optics were kept fixed to maintain a small probe beam $\left(r_{o}=2.5 \mu \mathrm{m}\right)$; the focusing lens in the pump arm was axially translated in order to vary the pump beam spot diameter and thus the acoustic source size, down to a diameter $2 r_{o}$ of about $6.5 \mu \mathrm{m}$.

In Fig. 3, we show the measured change in optical probe reflectivity $\Delta R$ induced by the picosecond acoustic wave forms which have propagated across the $485-\mu \mathrm{m} \mathrm{Si}$ wafer. Figure 3(a) is the signal measured with the pump beam defocused to give a waist of approximately $25 \mu \mathrm{m}$; in this case the probe transducer layer is in the acoustic near field of the source. Figure 3(b) is the signal obtained when the excitation beam waist radius is tightly focused, to about $r_{o}=3.25 \mu \mathrm{m}$; in this case diffraction-induced pulse reshaping is expected to occur. In general, the optical signal $\Delta R$ does not directly follow the strain field. Thus, the near-field signal $\Delta R_{\mathrm{nf}}(t)$ in Fig. 3(a) is not a simple $\tau e^{-\tau^{2} / 2}$ function, but rather is a convolution of the time-dependent strain field with the Altransducer-layer response function. ${ }^{3}$ Nevertheless, when the strain pulse propagates to the far field so its shape is that of the derivative of the near-field pulse, the observed optical signal $\Delta R_{\mathrm{ff}}(t)$ will also be reshaped and will appear as the derivative of the near-field pulse (i.e., $\Delta R_{\mathrm{ff}}(t)$ $\left.=\partial\left[\Delta R_{\mathrm{nf}}(t)\right] / \partial t\right)$. Thus, the Gouy phase shift is clearly manifested for picosecond ultrasonic pulses generated by a tightly focused laser beam.

While it is apparent from the data that the diffracted wave form approaches the temporal derivative of the source, there are additional effects that can be observed. The data shown in Fig. 3 display some ringing in the signal following the main pulse. This is most likely due to acoustic ringing in the pump transducer layer; this ringing also shows a $\pi / 2$ phase shift between the near- and far-field wave forms. Acoustic dispersion in the Si may also extend the temporal profile of the pulse, but for a propagation length of less than half of $1 \mathrm{~mm}$ these effects are expected to be small. ${ }^{10}$ Lastly, at high excitation power, nonlinear pulse propagation and soliton generation were observed very similar to those reported in. ${ }^{11}$ Thus, it was imperative for us to verify that the pulse-reshaping effects shown in Fig. 3 were due solely to diffraction and not due to acoustic nonlinearity. First, we investigated the pulse shape as a function of excitation power 


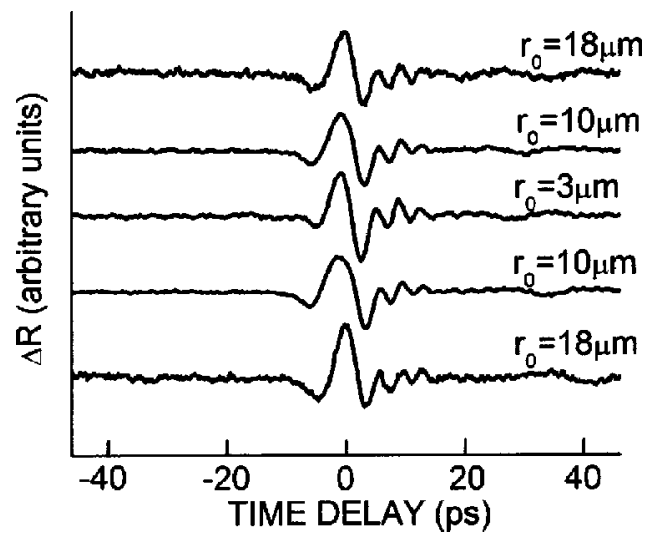

FIG. 4. Acoustic wave forms measured for various initial focusing conditions.

when the excitation beam was tightly focused (since it might be possible that the effect observed is due to a change in excitation power density as the spot size is changed, rather than diffraction). However, if the excitation power was kept sufficiently low, the pulse shape was found to be independent of power; this is the regime in which our experiments were performed.

Finally, in order to observe the continuous change in the acoustic pulse shape as a function of initial beam waist, we obtained a set of data over a range of pump focusing positions; the data are shown in Fig. 4. For an initial beam waist of $18 \mu \mathrm{m}(\zeta=0.04)$ the near-field acoustic pulse shape is observed. At $\zeta=1.08$, the shape is close to that expected for the far field. The smooth evolution in the shape for intermediate positions is evident. The ability to propagate picosecond ultrasonic pulses to the far field may be important in interpreting experiments on nonlinear propagation and dispersion effects with such pulses, and also opens up a range of potential applications in novel imaging schemes.

We thank H. J. Maris for assistance with numerical acoustic propagation algorithms. This work was supported by the National Science Foundation under grant STC PHY 8920108 and by the AFOSR under contract F49620-00-10328 through the MURI program.

${ }^{1}$ H. Grahn, H. Maris, and J. Tauc, IEEE J. Quantum Electron. 25, 2562 (1989).

${ }^{2}$ H. Maris, Sci. Am. 86, 278 (1998).

${ }^{3}$ C. Thomson, H. T. Grahn, H. J. Maris, and J. Tauc, Phys. Rev. B 34, 4129 (1986).

${ }^{4}$ A. B. Ruffin, J. Van Rudd, J. Decker, L. Sanchez-Palencia, L. LeHors, J. F. Whitaker, and T. B. Norris, IEEE J. Quantum Electron. 38, 1110 (2002).

${ }^{5}$ A. B. Ruffin, J. V. Rudd, J. F. Whitaker, S. Feng, and H. G. Winful, Phys. Rev. Lett. 84, 3410 (1999).

${ }^{6}$ S. Feng and H. G. Winful, Opt. Lett. 26, 485 (2001).

${ }^{7}$ J. W. Goodman, Introduction to Fourier Optics, 2nd ed. (McGraw-Hill, New York, 1996), Eq. 3-51.

${ }^{8}$ J. W. Goodman, op. cit., Eq. 3-57.

${ }^{9}$ A. E. Kaplan, J. Opt. Soc. Am. B 15, 951 (1998).

${ }^{10}$ H.-Y. Hao and H. J. Maris, Phys. Rev. B 63, 224301 (2001).

${ }^{11}$ H.-Y. Hao and H. J. Maris, Phys. Rev. B 64, 064302 (2001). 